Article

\title{
Software-Defined Radio Beamforming System for 5G/Radar Applications
}

\author{
Diogo Marinho $^{1,2, *}$, Raul Arruela ${ }^{1,2}$, Tiago Varum $^{1}$ (1) and João N. Matos ${ }^{1,2}$ (D) \\ 1 Instituto de Telecomunicações, Campus Universitário de Santiago, 3810-193 Aveiro, Portugal; \\ raul.marques@ua.pt (R.A.); tiago.varum@ua.pt (T.V.); matos@ua.pt (J.N.M.) \\ 2 Departamento de Eletrónica, Telecomunicações e Informática, Universidade de Aveiro, 3810-193 Aveiro, Portugal \\ * Correspondence: botelhomarinho@ua.pt
}

Received: 2 September 2020; Accepted: 10 October 2020; Published: 15 October 2020

\begin{abstract}
Based on the flexibility of software-defined radio (SDR) techniques applied to an array of antennas, this article presents a beamforming architecture designed to operate in millimeter-wave bands $(28 \mathrm{GHz})$, with possible applications in radar and $5 \mathrm{G}$ systems. The system structure, including its main constituents such as the radio frequency (RF) frontend modules, the radiating elements as well as the baseband processing on the host computer are widely described. Beamforming is achieved by digitally controlling the signals that feed the antennas. The experimental measurements performed in an anechoic chamber validate the proposed approach.
\end{abstract}

Keywords: digital beamforming; SDR; 5G; radar; beam shaping; phased array antenna

\section{Introduction}

The world is gradually undergoing the age of communications, facing an enormous density of traffic and connections, where everyone is online, people and objects, interacting with each other, through a multitude of applications [1]. The modern generation of mobile communications (5G) is launched, which brings new challenges and opportunities, and will allow the creation and integration of new networks such as the internet of things (IoT) and vehicular networks, in an increasing global coverage. These systems impose a set of new requirements such as high speed data transfer, low latency, high network capacity and high connections density [2], which involve the operation in a frequency range little used so far, in the zone of millimetre waves.

Radio detection and ranging (RADAR) technology, that was mainly used for military purposes, recently has suffered increasing attention, and its application is nowadays widespread in sports, biomedical, military or in transportation systems. Radars are used for surveillance, navigation, localization, traffic control or for weapons guidance. Currently, with the evolution of vehicular networks, smart cities and other intelligent systems, more advanced radar technologies are being developed. In the near future, the extension of the radar concept is expected for two purposes, reflectometry and communications. The scenario of Figure 1 is foreseeable, a panoply of interconnected systems sharing information, and experiencing a huge communications density, with high risk of interferences. 


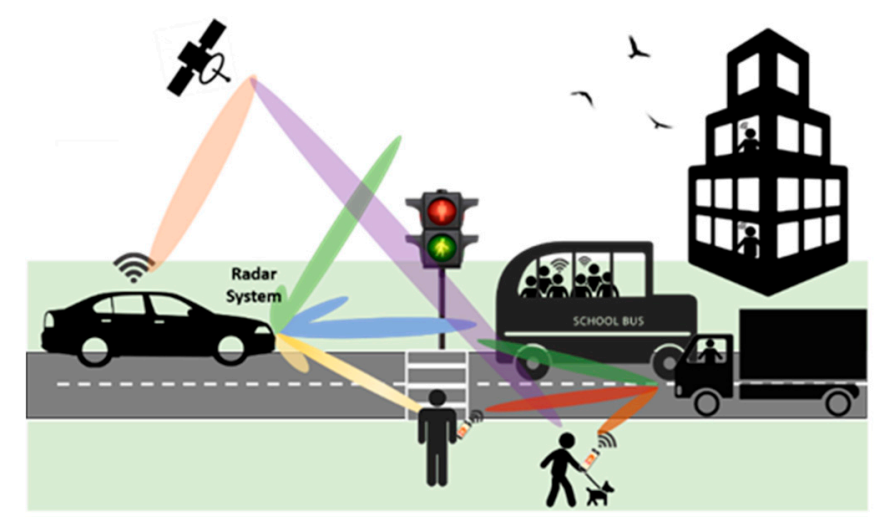

Figure 1. Modern environment of multicommunication systems.

Radar technology is required to filter the environment, focusing more accurately on the target or targets, as well as to mitigate interference signals from the surrounding environment. Multibeam capabilities, beamforming, high gain and tremendous versatility and reconfigurability are important aspects for modern radar systems. Additionally, most of these characteristics allow us to overcome propagation issues raised when these systems operate at higher frequencies. Thus, versatile and dynamic communication systems are needed, with the ability to continuously adjust to the applications in which they are inserted, maximizing the quality of communication. With additional signal processing capability, adaptive antennas use new digital architectures that, in real-time, dynamically adjust the radiation pattern of the array. These systems have perception of the received signal and optimize their radiation diagrams by changing the beam shape and beam direction, suppressing interferences by introducing nulls in the directions of the interference signals, changing the level of side lobes, compensating for hardware impairments and finally, accommodating the mutual coupling effects that can change the amplitude and phase of the transmitted signals. Particularly regarding automotive radars, the authors of [3,4] present a good example of the importance of using digital beamforming in multiantenna systems to reduce the power received in the directions of interference signals, placing nulls in the radiation pattern.

There have been technological advances in this field, where low-cost mm-wave radars composed of several antenna elements integrated in single board and with high resolution, have been presented. In [5] is presented an example of a low-cost digital beam steering receiver (Rx) phased array for IoT device connectivity, a cheap application to get over the communication problems. In [6], a frequency modulated continuous wave (FMCW) radar is described. This system generates multiple digital beams with high gain, low side lobe level, narrow beam width and high angular resolution control. The principles of the digital beamforming applied to synthetic aperture radar (SAR) systems are shown in [7], as well as the improvement in the resolution achieved regarding conventional radars.

In [8,9], a wide variety of future applications are shown, as well as the progress that technology is facing in reducing the cost of phased array antennas. Massive antenna arrays using up to 128 elements, increasing performance and gain of radar communication systems are presented in [10], taking advantage of digital beamforming based on coded aperture radar (CAR) technique at $77 \mathrm{GHz}$.

In [11-13], the importance of the beamforming spectral efficiency is demonstrated, with the cancelation of undesired interferences by placing nulls in their directions that can lead to a lower bit error rate (BER).

In [14], there is a software-defined phased array radio operating at $28 \mathrm{GHz}$ that uses software to control multiple beam characteristics. However, additional hardware was required, a field programmable gate array (FPGA) board for digital control and an Ettus B200 mini software-defined radio (SDR) for data waveform control. In [15], a highly compact $28 \mathrm{GHz}$ complementary metal-oxide-semiconductor (CMOS) integrated circuit (IC) with multiple input multiple output (MIMO) and beamforming capabilities with a single wire for baseband multiplexing is reported. 
However, it requires heavy digital signal processing (DSP) MIMO operations to retrieve the information. A similar concept was recently shown in [16], proposing a digital array receiver for satellite applications, also integrating DSP algorithms, operating in a single-chip RF system on chip (SoC) solution installed on the Xilinx ZCU1275 prototyping platform; however, operating in a fixed frequency range between 27.5 and $28.35 \mathrm{GHz}$. A 64-channel massive MIMO transceiver with a fully digital beamforming (DBF) architecture is presented in [17]. The MIMO transceiver operates in time division duplex (TDD) mode, at $28 \mathrm{GHz}$ with a $500 \mathrm{MHz}$ signal bandwidth. A high data rate in the communication was achieved using the beam-tracking technique and two streams of 64-QAM (Quadrature Amplitude Modulation) signals. However, one of the great difficulties of this architecture is the high cost of the hardware structure that supports the 64 channels. Due to the high power consumption of the RF frontends, additional space in the circuit is required for heat dissipation. A Ka band DBF array transmitter using a direct digital synthesizer (DDS) for millimeter-wave applications is proposed in [18], operating at $24 \mathrm{GHz}$. This system reveals a complex calibration and has fluctuation over different scanning angles that unbalance the gain of different beam peaks in the $1 \times 15$ antenna array. In [19], the implementation of an $\mathrm{X}$ band $4 \times 4$ digital phased array module is shown, which includes one custom CMOS RF SoC. This system has high side lobes due to the transmitter $(\mathrm{Tx})$ calibration process that involves random errors both in the phase and magnitude.

The concept of beamforming, in the sense of the ability to control the radiation shape of an antenna array, can be performed either digitally, like the examples presented previously [14-19], analogically (by using the frontend analog phase shifters [20]) or in a hybrid way [21]. A millimeter-wave hybrid beamforming system architecture based on analog phased subarray is reported in [22]. This work uses a 32-element antenna array with a hybrid beamforming transceiver to operate at the $28 \mathrm{GHz}$ band in TDD mode, with $500 \mathrm{MHz}$ bandwidth. An important aspect is the reduction in the number of intermediate frequency (IF) channels compared to [14-19], reducing the cost comparing to fully digital architectures. However, one issue with this architecture is the analog phase shifting, which is critical to increasing the insertion loss up to $19.5 \mathrm{~dB}$, requiring the use of additional amplifiers to compensate such losses. Additionally, this architecture has a higher complex design of the control signal because of the non-linearity between the control signals (amplitude weighting control) and output phase of this vector-sum phase. Authors in [23] explored the use of a Universal Software Radio Peripheral (USRP) X310 connected to a computer capable of calculating the direction of arrival (DoA) and generating beamforming in the sub- $6 \mathrm{GHz}$ frequency band for a satellite transponder structure. Nevertheless, this test bed presents considerable DoA estimation errors in a lower frequency of the operation scenario.

Millimeter-wave RF systems are evolving rapidly, to some extent supported by software-defined radios. These systems provide a straightforward way to rapidly interact with hardware, and to test their use. Therefore, the combination of digital technology and versatile architectures, with adaptive antenna processing techniques, allows for powerful systems that can be used in communications and radar approaches.

In this work, a versatile and reconfigurable $4 \times 4$ SDR digital beamforming phased array system operating in the Ka band at $28 \mathrm{GHz}$ is proposed. This system has the capability of electronically and digitally manipulating and steering the radiation pattern of an antenna array, and adjusting it to the environment, maximizing its performance and efficiency. Besides that, this system also has the versatility to, with low complexity, change the frequency of the local oscillator (LO) and the IF to implement communication systems and radar in the millimeter waves (Ka band), enabling the rapid testing of new solutions based on software-defined radio. It has also the advantage of carrying out full-duplex communication. This architecture has the potential to be used in a great variety of future scenarios such as in 5G communications, radar applications, IoT or surveillance. It is an extension work regarding the previous papers $[24,25]$ with improvement in the digital processing unit and practical measurements validation. 
This paper is organized into five sections, starting with the Introduction in Section 1, stating the objectives and providing a brief state-of-the-art on SDR beamforming systems. Section 2 presents both digital and analog components that constitute the proposed architecture of the software-defined radio (SDR) phased array system. Section 3 describes the beamforming processing system while in Section 4 the experimental setup and the measurement results are presented. Finally, the last section reports the main conclusions of the work.

\section{System Architecture}

Figure 2 shows the proposed architecture of the $28 \mathrm{GHz}$ SDR system, using a digital beamforming phased array structure. This architecture has two complementary domains, the digital and the analog, which are described below.

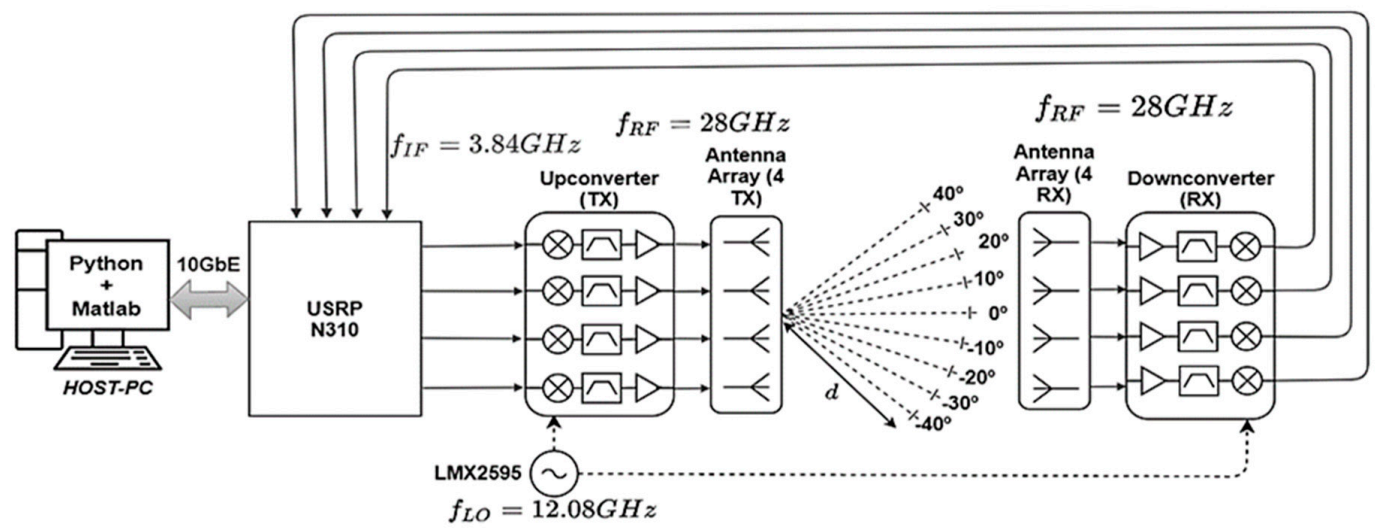

Figure 2. Block diagram of the proposed SDR beamforming system.

\subsection{Digital Component}

The digital component of the proposed system includes one Ettus SDR Universal Software Radio Peripheral (USRP) N310 from National Instruments and one Host-PC (Personal Computer). The USRP is a network SDR equipment (uses gigabit ethernet interface) which has four independent transmitting and receiving channels, operating at a frequency that can be chosen from $10 \mathrm{MHz}$ up to $6 \mathrm{GHz}$, with up to $100 \mathrm{MHz}$ of bandwidth. This equipment is responsible for digitally generating any required signal for the desired application and modulating it with a RF microwave carrier. The baseband signal is digitally decomposed into two orthogonal components that feed a phase and quadrature modulator. The block diagram of the signal modulation performed inside the USRP is shown in Figure 3. At the USRP output, the resulting modulated intermediate frequency (IF) signal is chosen to have a $3.84 \mathrm{GHz}$ carrier frequency $(\mathrm{fc})$. An equivalent reverse process is applied to the IF reception signals. The received IF signal is demodulated in phase and quadrature, generating I/Q samples that are later sent out over a $10 \mathrm{GbE}$ connection to the HOST-PC for processing. This equipment is controlled by the HOST-PC, where a Python script based on the USRP hardware drivers (UHD) library was developed.

The N310 architecture is divided into two segments, as shown in Figure 3: a pair of daughterboards, each based on the Analog Devices AD9371 transceiver, and one motherboard composed by one Arm Cortex A9 and one FPGA (Kintex-7). In this work, all the core digital signal processing is performed in the USRP from the selection of frequency of the local oscillator, the sample rates, decimation/interpolation and the selection of appropriate filter bandwidth. The remaining processing of the In-phase and Quadrature (IQ) samples is performed on the PC. Regarding the communication between the HOST-PC and the USRP, the UHD library used in the PC to control USRP parameters is illustrated in Figure 4, which provides a set of important functions for the communication. Figure 4 focuses with high detail on the block diagram of the software structure developed on the PC to calibrate the system and manage the beamforming. A Python program adapted to the USRP N310 was created, to control the generated and received waveform signals. In this script, eight threads were created, each one 
independently controlling each channel of the USRP. The process starts by requesting samples from the USRP, encoding them with the channel number and sending them over the Transmission Control Protocol/Internet Protocol (TCP/IP) socket. In this case, the Matlab program is the client, which for each channel decodes the samples in an IQ vector. A calibration routine was also developed, calculating the phase and amplitude differences existing among the RF frontend channels.

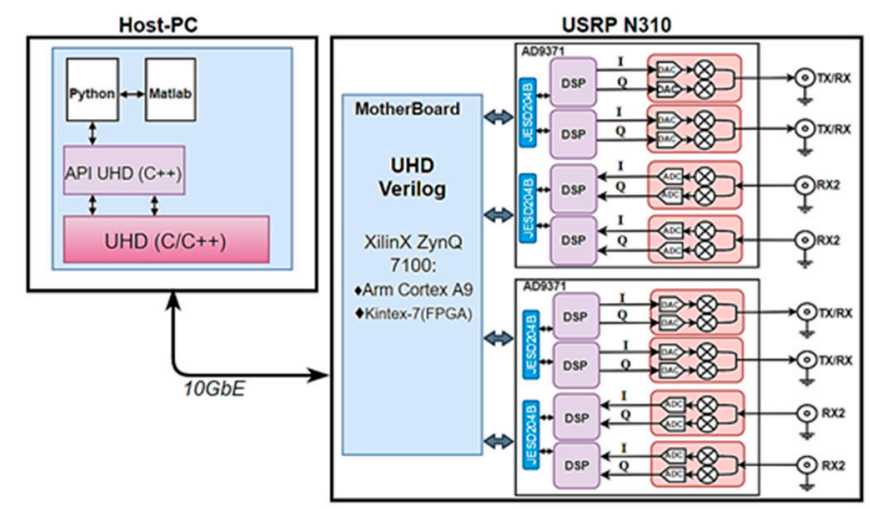

Figure 3. Block diagram of software interface between Host-PC and Universal Software Radio Peripheral (USRP) N310.
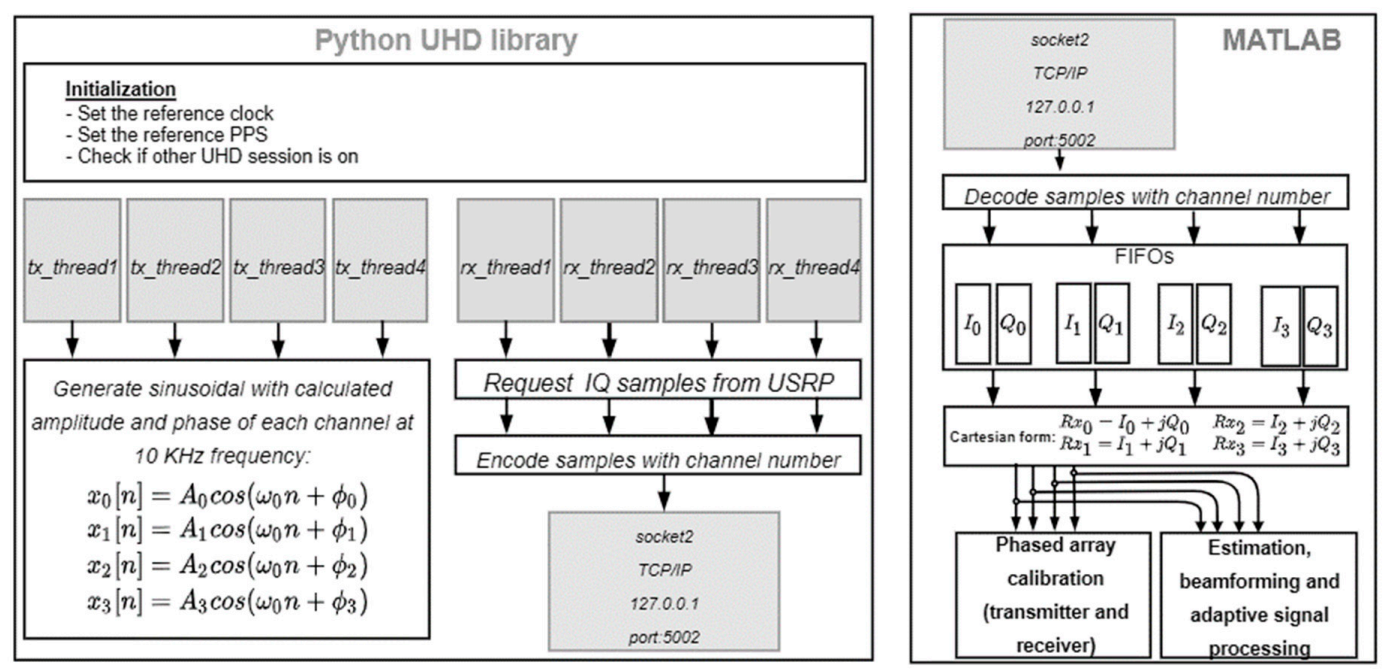

Figure 4. Flow control between the receiver and transmitter during Python and Matlab interfaces.

\subsection{Analog Component}

The analog component of the proposed system is a transceiver composed of frequency converter modules, between IF and RF, and also by the antenna arrays that transmit and collect the involved signals. The operating frequency was selected in the Ka band, at $28 \mathrm{GHz}$, and the chosen IF was 3.84 GHz.

Therefore, the RF upconverter module translates the modulated $3.84 \mathrm{GHz}$ IF signal to the output operating frequency of $28 \mathrm{GHz}$. The block diagram of the upconverter is presented in Figure 5a, and its measurements are shown in Figure 6. The RF downconverter transforms the received $28 \mathrm{GHz}$ signals down to $3.84 \mathrm{GHz}$ IF, to be processed in the USRP. The block diagram of the downconverter can be seen in Figure $7 \mathrm{a}$, and its measured results are provided in Figure 8.

\subsubsection{RF Frontend Modules}

USRP channels can operate with frequencies up to $6 \mathrm{GHz}$ (max), so to allow the system to operate at $28 \mathrm{GHz}$, frequency conversion units are required to be attached to the USRP channels, connected to 
the antennas. Therefore, two RF frontend units were developed, allowing this conversion from 3.84 to $28 \mathrm{GHz}$ and vice versa.

The chosen topology uses the heterodyne configuration. The upconverter is composed, in each channel, by an active mixer, a bandpass filter and an amplifier. To distribute the signal from the local oscillator (LO) to each channel mixer, discrete power splitters were also used.

The key element for the up/down conversion is the mixer. The chosen mixer was the HMC264LC3B from Analog Devices. It is a subharmonic mixer that operates from 21 to $31 \mathrm{GHz}$ at the RF port, using a signal from Direct Current (DC) to $6 \mathrm{GHz}$ at the IF port, and from 10.5 to $15.5 \mathrm{GHz}$ at the $\mathrm{LO}$ port.

The used amplifier is the MAAL-011129 from MACOM, which is a low noise broadband amplifier (18 to $31.5 \mathrm{GHz}$ ) with a gain of $18 \mathrm{~dB} @ 28 \mathrm{GHz}$ [19]. The local oscillator signal is generated by the phased locked loop (PLL) LMX2595 evaluation board which operates over a wideband (from $10 \mathrm{MHz}$ up to $19 \mathrm{GHz}$ ) with low noise characteristics. The power splitter that divides the $\mathrm{LO}$ into two channels is the Mini-Circuits EP2k1+, which works from 1.8 to $28 \mathrm{GHz}$. The selected LO was $12.08 \mathrm{GHz}$. Finally, a fifth order parallel coupled microstrip band pass filter was designed with $5 \mathrm{GHz}$ of bandwidth, an attenuation of approximately $40 \mathrm{~dB}$ at the image frequency $(20 \mathrm{GHz})$ and $0.9 \mathrm{~dB}$ of insertion loss at $28 \mathrm{GHz}$.

The upconverter module comprises four IF inputs, four RF outputs and two LO inputs. It was designed for a maximum power of about $10 \mathrm{dBm}$ at the RF outputs, and for a maximum IF input power of $7 \mathrm{dBm}$, resulting in an expected conversion gain of $3 \mathrm{~dB}$.

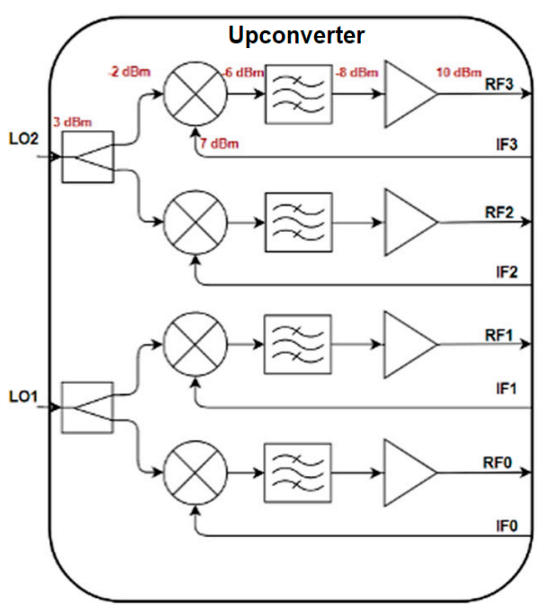

(a)

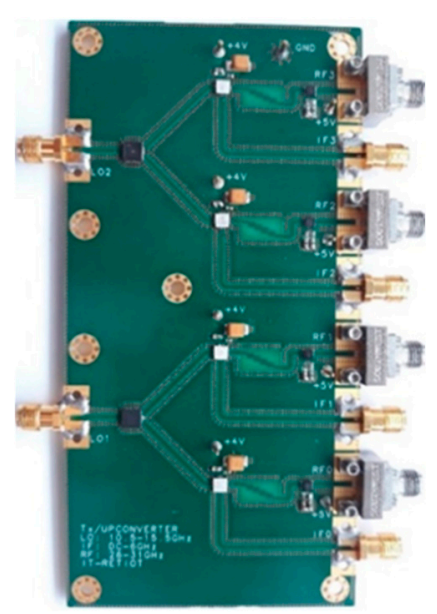

(b)

Figure 5. Upconverter designed module: (a) block diagram, (b) fabricated prototype board.

The purpose of the designed filter is to mitigate the image frequency signal and other spurious signals generated by the mixer. It is assumed that the IF signals at the inputs are bandpass at $3.84 \mathrm{GHz}$ with the proper filtering done in the USRP. A photograph of the fabricated prototype can be seen in Figure 5b).

The upconverter was tested using a $12 \mathrm{GHz}$ signal in the LO port and with an IF of $4 \mathrm{GHz}$. Figure 6a shows the output spectrum at one of the four RF outputs of the upconverter. It is possible to find out the image signal $(24-4 \mathrm{GHz}=20 \mathrm{GHz})$, the LO signal $(2 \times 12 \mathrm{GHz})$ and the desired RF signal $(24+4 \mathrm{GHz}=28 \mathrm{GHz})$. The behaviour of the filter can also be observed, since both the image and LO signals are highly attenuated compared to the RF signal, 28.8 and $26.4 \mathrm{dBm}$, respectively.

Figure $6 \mathrm{~b}$ presents the measured conversion gain of the upconverter at different $\mathrm{RF}$ frequencies. It should be noted that the RF variation was made through the $\mathrm{LO}$. The measured gain was $-1 \mathrm{~dB}$ at $28 \mathrm{GHz}$, slightly below the expected $3 \mathrm{~dB}$. Regarding the $1 \mathrm{~dB}$ compression point (P1dB) at the output, $8.6 \mathrm{dBm}$ was obtained, a value relatively close to the expected $(10 \mathrm{dBm}$, as can be seen on the link power budget in Figure 5a). 


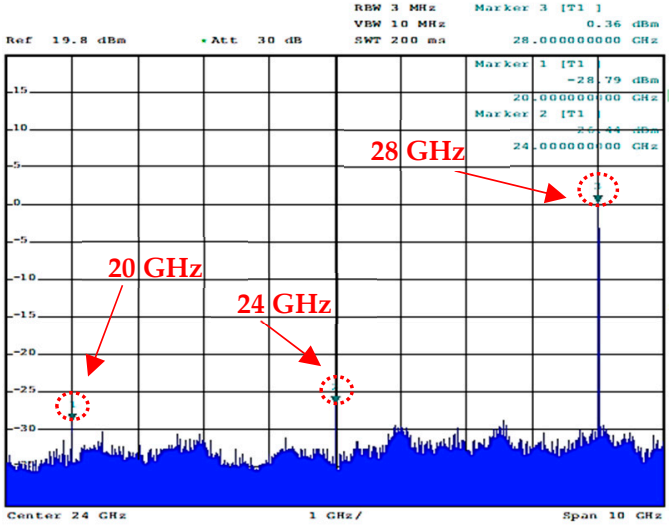

(a)

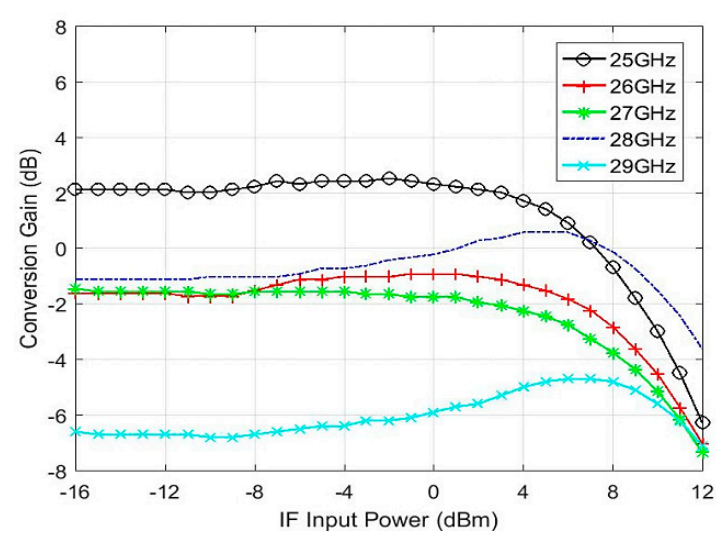

(b)

Figure 6. Measurements of upconverter module: (a) RF output spectrum (M1@20 GHz, M2@24 GHz, M3@28 GHz; (b) upconverter conversion gain at different RF frequencies.

The downconverter module was designed similarly as the upconverter. The purpose of the filter is to reject any image signal that can be captured at the RF inputs. In this structure, the mixer is the element that imposes the maximum power on the RF inputs since it has a P1dB compression point of $3 \mathrm{dBm}$. Thus, the maximum RF input power will be $-13 \mathrm{dBm}$ and the maximum power at the IF output will be $-10 \mathrm{dBm}$, resulting in an expected conversion gain of $3 \mathrm{~dB}$. The photograph of the fabricated prototype is shown in Figure $7 \mathrm{~b}$.

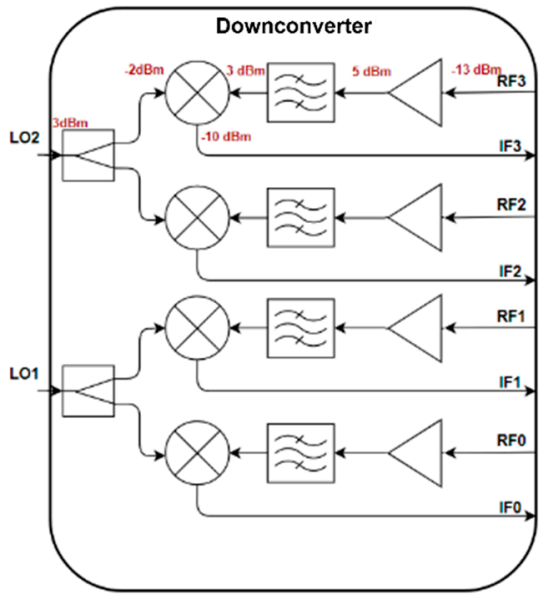

(a)

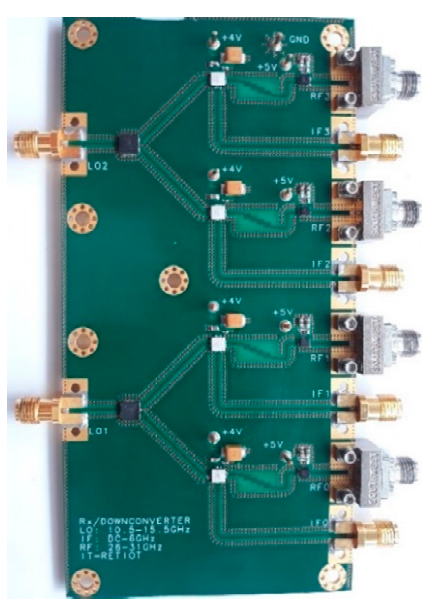

(b)

Figure 7. Downconverter designed module: (a) block diagram; (b) fabricated prototype board.

In Figure 8a is shown the output spectrum at one IF port of the downconverter module. The measurement test was performed using an LO of $12 \mathrm{GHz}$ and RF signal of $28 \mathrm{GHz}$. Marker M1 is at the desired IF frequency, $4 \mathrm{GHz}(28-24 \mathrm{GHz})$ and M3 is at the LO frequency signal $(12 \mathrm{GHz})$. The downconverter accomplished a conversion gain of $4 \mathrm{~dB}$, too close to the expected $3 \mathrm{~dB}$. As for the $\mathrm{P} 1 \mathrm{~dB}$ at the RF input, $-7 \mathrm{dBm}$ was measured, a value well above the expected $-13 \mathrm{dBm}$. The third order intersection point is $4.4 \mathrm{dBm}$, and the intermodulation ratio is $39.2 \mathrm{~dB}$.

As in the upconverter, the results suggest a shift in the central frequency of the mixer, since the results at $25 \mathrm{GHz}$ are better than at $29 \mathrm{GHz}$. In any case, at the frequency of interest, the results are quite satisfactory and close to what was intended. 


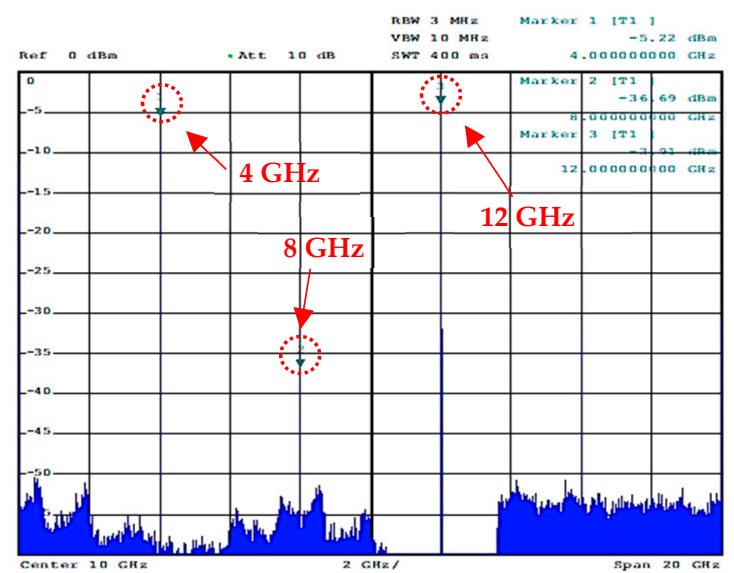

(a)

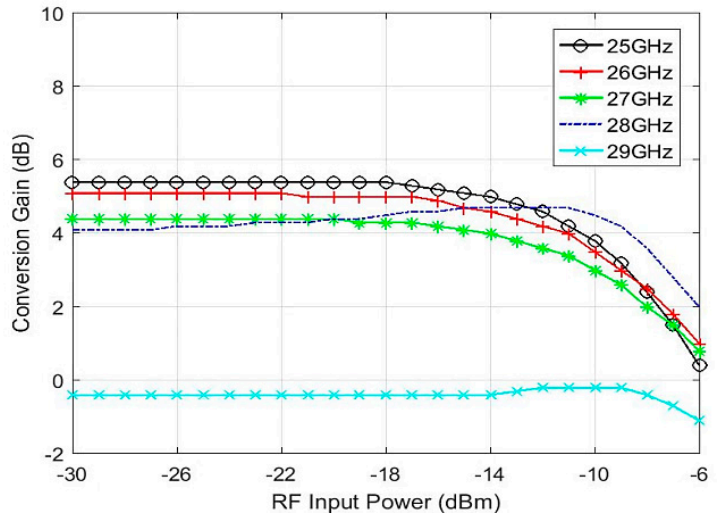

(b)

Figure 8. Measurements of downconverter module: (a) RF output spectrum (M1@4 GHz, M3@12 GHz; (b) downconverter conversion gain at different RF frequencies.

\subsubsection{Planar Antenna Array $4 \times 4$}

The antenna used at the interface between the free space and the RF frontend modules operates at $28 \mathrm{GHz}$ and is a $4 \times 4$ series feed planar antenna array described in [26] and shown in Figure 9 . The antenna consists of $\mathrm{N}=4$ individual series feed linear arrays, each using four microstrip patches. The array can provide control of the radiation pattern in the horizontal plane. In [26] is shown the simulated radiation pattern on the horizontal axis. It is possible to observe that the radiation pattern can be steered in different directions by applying a progressive phase shift in the feeding of each subarray. The gain decreases progressively when the direction of radiation moves out of the boresight.

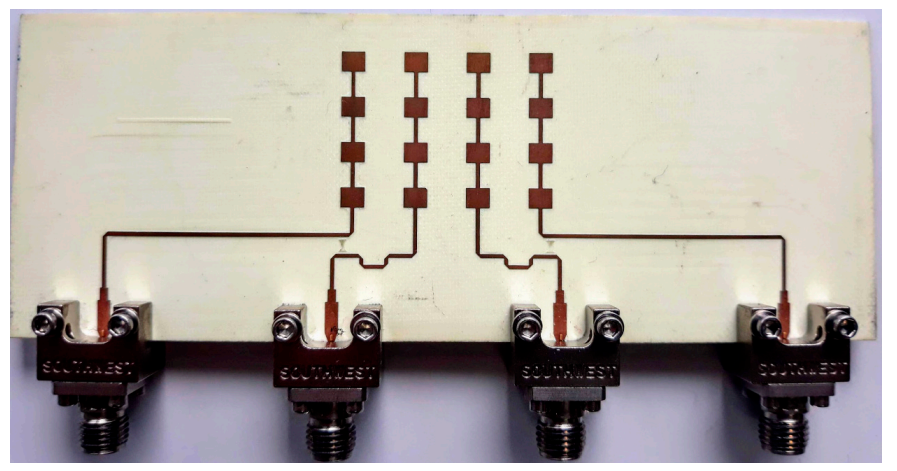

Figure 9. Planar antenna array $4 \times 4$.

\section{Beamforming Processing}

In the previous section, the architecture of the SDR system was presented, as well as the description of each analog and digital component used. In this section, taking advantage of the absolute control over each of the four $\mathrm{Tx} / \mathrm{Rx}$ channels and the processing capacity that this system has, an example demonstrating some of its potentialities is presented. In this work, some examples will be presented using two possibilities, beam shaping and beam steering, to highlight the functionality of the proposed system. Nevertheless, a multitude of techniques may still be explored in the future. Figure 10 shows a block diagram of a beamforming system, applied to a linear array with $\mathrm{N}$ number of isotropic elements, in reception. In this structure, each antenna element has an adjustment, by multiplying each received signal by a weight factor, $W_{n}=a_{n} e^{j \alpha}$, where $a_{n}$ is the amplitude excitation, and $\alpha$ is the relative phase excitation to the previous element. By varying the weight factor of each channel, and properly estimating its values, it is possible to control the radiation pattern of the array. 


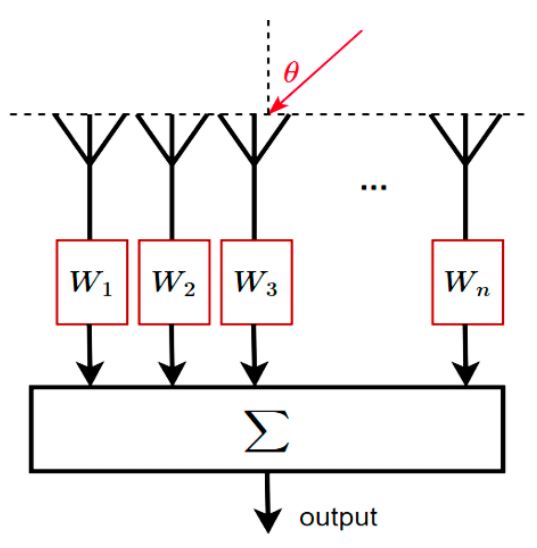

Figure 10. Block diagram of Rx antenna array.

The far field of the array factor can be written according to the Equation (1), which relates the contribution of each element for the total radiation of a linear array.

$$
A F=\sum_{n=1}^{N} a_{n} e^{j[(n-1) k d \cos \theta+\alpha]}
$$

In Equation (1), the summation of independent phasors (weights), the progressive phase is represented by $\psi=k d \cos \theta+\alpha$, where $k=(2 \pi \lambda)$ and $d$ the distance between elements of the array.

\subsection{Beam Steerin}

Considering an array of $\mathrm{N}=4$ elements, with equal and unitary excitation amplitude for all elements, $a_{n}=1$, the respective phase delay to apply to each channel in order to have the array steer its radiation to the position $\theta$ is given by $\alpha=-k d \cos \theta$. Table 1 shows the estimated phase delays for each channel, considering the set of chosen locations $(\theta)$, using an array of half-wavelength spaced elements.

Table 1. Beamforming phase weights.

\begin{tabular}{ccccc}
\hline Steering Angle $\boldsymbol{\theta}\left({ }^{\circ}\right)$ & $\alpha_{1}\left({ }^{\circ}\right)$ & $\alpha_{2}\left({ }^{\circ}\right)$ & $\alpha_{3}\left({ }^{\circ}\right)$ & $\alpha_{4}\left({ }^{\circ}\right)$ \\
\hline $40^{\circ}$ & $0^{\circ}$ & $173^{\circ}$ & $346^{\circ}$ & $519^{\circ}$ \\
$30^{\circ}$ & $0^{\circ}$ & $135^{\circ}$ & $270^{\circ}$ & $405^{\circ}$ \\
$20^{\circ}$ & $0^{\circ}$ & $92^{\circ}$ & $184^{\circ}$ & $276^{\circ}$ \\
$10^{\circ}$ & $0^{\circ}$ & $47^{\circ}$ & $94^{\circ}$ & $141^{\circ}$ \\
$0^{\circ}$ & $0^{\circ}$ & $0^{\circ}$ & $0^{\circ}$ & $0^{\circ}$ \\
$-10^{\circ}$ & $0^{\circ}$ & $-47^{\circ}$ & $-94^{\circ}$ & $-141^{\circ}$ \\
$-20^{\circ}$ & $0^{\circ}$ & $-92^{\circ}$ & $-184^{\circ}$ & $-276^{\circ}$ \\
$-30^{\circ}$ & $0^{\circ}$ & $-135^{\circ}$ & $-270^{\circ}$ & $-405^{\circ}$ \\
$-40^{\circ}$ & $0^{\circ}$ & $-173^{\circ}$ & $-347^{\circ}$ & $-519^{\circ}$ \\
\hline
\end{tabular}

\subsection{Beam Shaping}

The concept of beam shaping is vast and brings together all the techniques that, through feeding of the array, allows the modification of its radiation shape. Typically, this concept is used in communications to mitigate the influence of interfering signals through side lobes (reducing or eliminating them) or confining the width of the main lobe.

There are different reported methods in the literature, such as the Schelkunoff polynomial method, minimum mean-square error (MMSE) weight and many others, and in this work, two methods, Dolph-Tschebyscheff and binomial [27], were applied in the proposed digital system and tested to demonstrate their impact on the radiation of the antenna array when compared to the uniform array. 
Using the theoretical expressions of [27], for the array of $\mathrm{N}=4$ elements, the feeding distributions were estimated for the binomial method and for the Dolph-Tschebyscheff technique using different values of side lobe level (SLL), such as $-15 \mathrm{~dB},-20 \mathrm{~dB}$ and $-25 \mathrm{~dB}$. The SLL represents the signals being radiated in the unwanted direction. Applying such methods in practical application could be very helpful to reduce the interferences.

These distributions are provided in Table 2 and were applied to the theoretical Equation (1). In the Matlab, the different radiation patterns, considering all the techniques, were calculated using (1) and multiplying by the theoretical beam pattern of a microstrip patch antenna (element factor), which is the radiating element chosen. The results are shown in Figure 11.

Table 2. Estimated amplitude distribution.

\begin{tabular}{ccccc}
\hline Weight Method & a1 & a2 & a3 & a4 \\
\hline Uniform & 1 & 1 & 1 & 1 \\
Binomial & 1 & 3 & 3 & 1 \\
Dolph-Tschebyscheff $(-15 \mathrm{~dB})$ & 1 & 1.33 & 1.33 & 1 \\
Dolph-Tschebyscheff $(-20 \mathrm{~dB})$ & 1 & 1.74 & 1.74 & 1 \\
Dolph-Tschebyscheff $(-25 \mathrm{~dB})$ & 1 & 2 & 2 & 1 \\
\hline
\end{tabular}

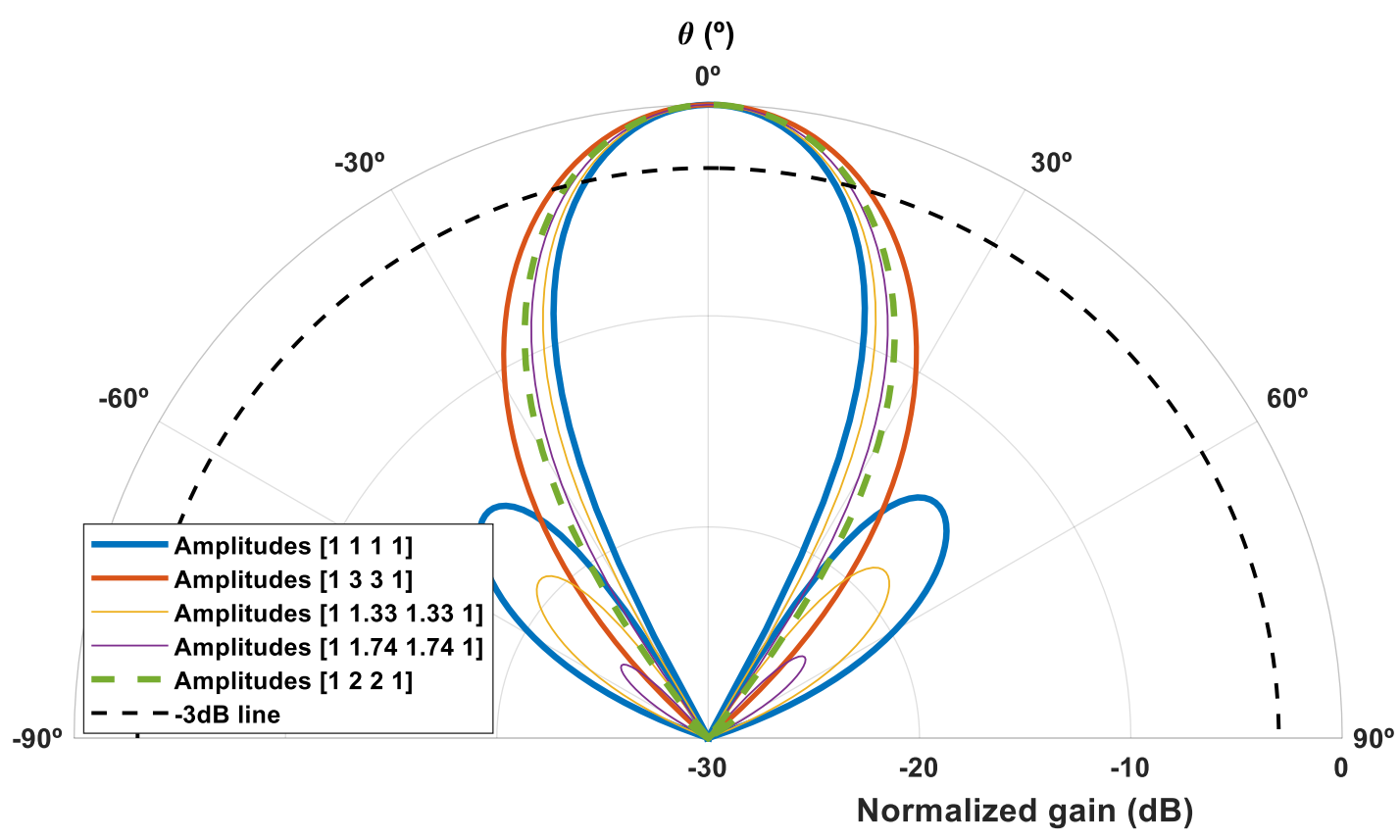

Figure 11. Theoretical radiation patterns considering the distribution weights.

According to Figure 11, it is possible to observe several aspects resulting from the use of each method. First, using the uniform distribution, with all elements fed with equal amplitude and phase, a higher side lobe is observable, and the amplitude of this lobe reduces with the application of the different methods, namely using the Dolph-Tschebyscheff technique (for the different SLL estimations), and in particular using the binomial method, for which the side lobes even disappear. It can also be proved that the amplitude distribution from the application of the Dolph-Tschebyscheff method for very small SLL values will tend towards the binomial distribution.

Another important aspect that can be taken from Figure 11 is the variation of the half power beam width (HPBW), using the different methods, observing that this value increases as the value of the SLL decreases.

The standard definition of beam width is the angle aperture from which most of the power is radiated from antennas [27]. It is a very important characteristic of an antenna array, and a 
crucial characteristic in phased array antennas and beamforming, because of the useful advantage of minimizing unwanted interference signals by controlling the main lobe characteristics, beam width and the side lobes.

The concept of half power beam width (HPBW) is commonly used and reflects the angular aperture in which the gain of the antenna falls $3 \mathrm{~dB}$. There are theoretical expressions reported in the literature [27] that estimate this value.

\section{Measurement Setup and Results}

The measurements scenario of the proposed system is illustrated in Figure 12. The setup was placed in an anechoic chamber, and included a power supply, providing the DC voltage to the RF up/downconverter's boards and the PLL LMX2595 (LO frequency of $12.08 \mathrm{GHz}$ ). In addition to the frontend boards and the PLL, the setup also included the Host-PC, the Tx and Rx antenna arrays, and the USRP N310, as can be identified in the Figure 12.

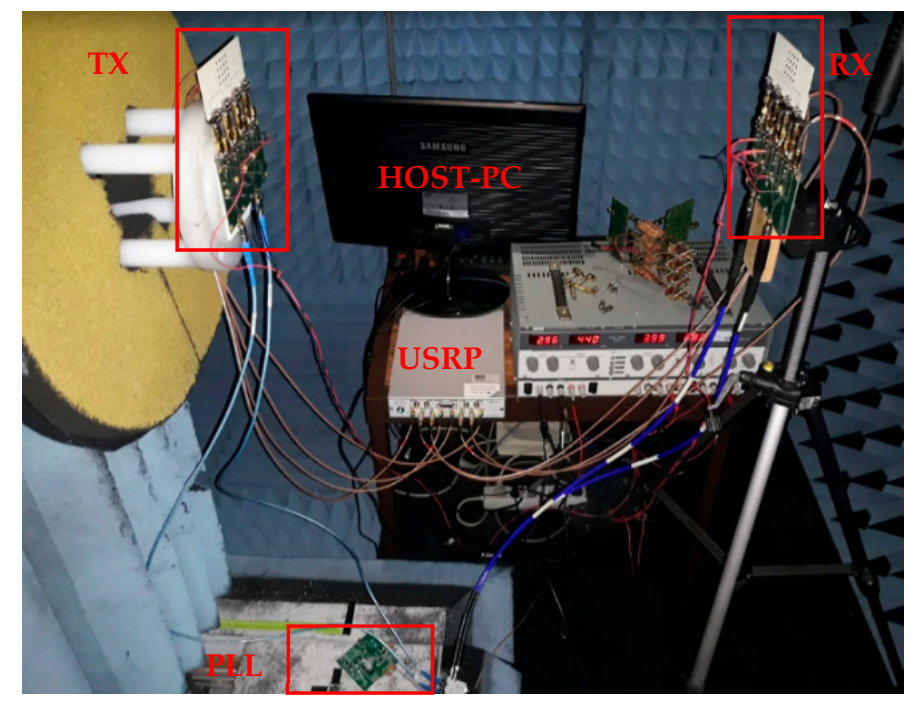

Figure 12. Experimental setup to measure antenna radiation pattern.

Before starting the measurement process, and after carefully placing all the elements of the setup, the first task was to calibrate the system, in order to ensure that all signals reached the antennas in phase, and all signals received maintained their properties until they were read by the USRP. The principle of calibration is the same as beamforming, and consists of adding certain weight (phase and amplitude) to each channel with the purpose of compensating for the hardware impairments in the upconverter/downconverter modules, the phase differences in the USRP daughterboard and the temperature drifts. Using the Python/Matlab interface, a calibration routine was developed to compensate for the phase and amplitude differences of the channels.

The four elements of the transmitter phased array antenna were placed in the rotor arm of the anechoic chamber, $65 \mathrm{~cm}$ away from the receiver (ensuring the far field distance). In Matlab/Python, a function that calculates the correct phase to apply to each channel based on the desired direction of beamforming $\theta\left(^{\circ}\right)$ was developed. The USRP modulated the baseband signal into two orthogonal carrier waves, thus generating a $3.84 \mathrm{GHz}$ IF signal. This signal was then applied to an upconverter module that converted it to $28 \mathrm{GHz}$. The resultant signal was fed into one element of the antenna array. Since there is a channel digitally controlled (in amplitude and phase) for each element of the array, it is possible to use various beamforming algorithms. In the tests carried out, the radiation beam varied (in relation to the perpendicular plane of the array) between $-40^{\circ}$ and $+40^{\circ}$ with one degree increments. This procedure was repeated for nine different positions of the transmitter. For each step, the received signal in the antennas were captured, downconverted to $3.84 \mathrm{GHz}$, and then the IQ demodulation 
was performed in the USRP to obtain the IQ samples. From these received samples, it was possible to measure the amplitude and phase of the signal coming from each channel.

The first measurement using the system was related to beam steering, in which the phases estimated in Table 1 were applied to the four channels, to steer the beam to different angles. Figure 13a shows the normalized measured radiation patterns of the received signal for nine different positions of the transmitter, using uniform amplitude in the feeding of the array.

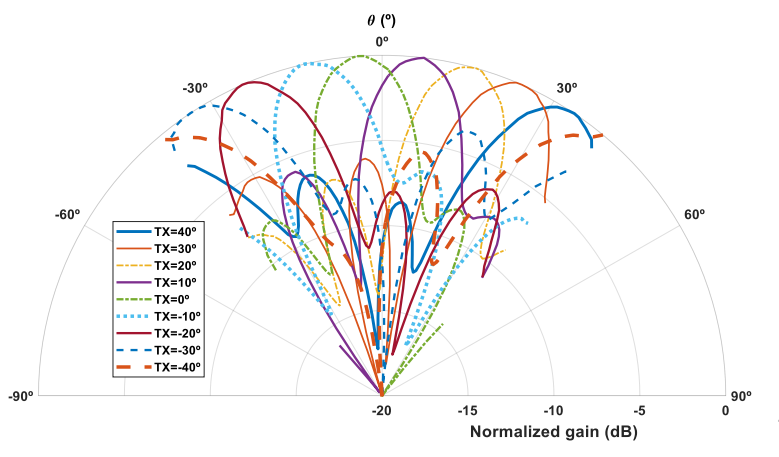

(a)

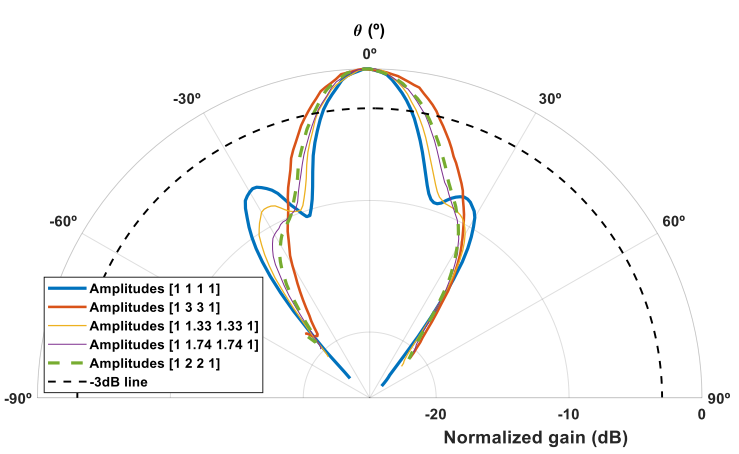

(b)

Figure 13. Measurement results: (a) polar plot of the measured radiation patterns; (b) measured antenna radiation pattern for many amplitude variations.

The second measurement was devoted to the beam width of the radiation pattern. It is possible to observe good agreement between the estimated curves of the array factor (Figure 11) and the measured values of the radiation pattern of Figure 13b. It is noticeable that the radiation pattern with the widest beam is the one that uses the binomial distribution $\left(27^{\circ}\right)$ and the narrowest beam is related with those that use uniform distribution $\left(17^{\circ}\right)$.

Table 3 compares the information of the measured beam widths of radiation patterns from Figure $13 b$ and those taken from the theoretical results of Figure 11. It is possible to verify good correspondence between the evolution of these values.

Table 3. Half power beam width (HPBW), measured and simulated.

\begin{tabular}{ccc}
\hline Weight Method & Beam Width Measured $\left(^{\circ}\right)$ & Beam Width Theoretical $\left(^{\circ}\right)$ \\
\hline Uniform & $17^{\circ}$ & $24^{\circ}$ \\
Binomial & $27^{\circ}$ & $32^{\circ}$ \\
Dolph-Tschebyscheff $(-15 \mathrm{~dB})$ & $19^{\circ}$ & $26^{\circ}$ \\
Dolph-Tschebyscheff $(-20 \mathrm{~dB})$ & $21^{\circ}$ & $28^{\circ}$ \\
Dolph-Tschebyscheff $(-25 \mathrm{~dB})$ & $22.5^{\circ}$ & $30^{\circ}$ \\
\hline
\end{tabular}

\section{Conclusions}

In this work, a $28 \mathrm{GHz}$ SDR beamforming antenna system was proposed for 5G, radar or IoT applications. All the analog and digital modules were properly developed and described, allowing us to obtain a system operating in the Ka band. The use of USRP enabled a flexible and versatile architecture since all its characteristics could be adjusted digitally. The experimental results have shown good agreement between the simulated and measured radiation patterns. It was possible to verify techniques to shape the beam of the array, as well as steering it, in a simple way, through the control of the signal amplitude and phase of each channel in the USRP. This system demonstrates great flexibility and scalability to be used in a wide range of equipment, especially in radar, mobile and satellite communication systems. 
Author Contributions: Conceptualization, D.M., R.A., T.V. and J.N.M.; methodology, D.M., R.A. and T.V.; software, D.M., and R.A.; validation, D.M., R.A., T.V. and J.N.M.; formal analysis, D.M., and R.A.; investigation, D.M., R.A. and T.V.; resources, T.V. and J.N.M.; data curation, D.M.; writing-original draft preparation, D.M., R.A. and T.V.; writing-review and editing, T.V. and J.N.M.; visualization, T.V.; supervision, T.V. and J.N.M.; project administration, J.N.M.; funding acquisition, J.N.M. All authors have read and agreed to the published version of the manuscript.

Funding: This work was partially supported by FCT/MCTES through national funds and when applicable cofunded EU funds under the project UIDB/50008/2020-UIDP/50008/2020 and the European Regional Development Fund through the Competitiveness and Internationalization Operational Program, Regional Operational Program of Lisbon, Regional Operational Program of the Algarve, in component FEDER, and the Foundation for Science and Technology, Project RETIOT, POCI-01-0145-FEDER-016432.

Conflicts of Interest: The authors declare no conflict of interest.

\section{References}

1. Hong, W.; Jiang, Z.H.; Yu, C.; Zhou, J.; Chen, P.; Yu, Z.; Zhang, H.; Yang, B.; Pang, X.; Jiang, M.; et al. Multibeam Antenna Technologies for 5G Wireless Communications. IEEE Trans. Antennas Propag. 2017, 65, 6231-6249. [CrossRef]

2. Shafi, M.; Molisch, A.F.; Smith, P.J.; Haustein, T.; Zhu, P.; De Silva, P.; Tufvesson, F.; Benjebbour, A.; Wunder, G. 5G: A Tutorial Overview of Standards, Trials, Challenges, Deployment, and Practice. IEEE J. Sel. Areas Commun. 2017, 35, 1201-1221. [CrossRef]

3. Bechter, J.; Rameez, M.; Waldschmidt, C. Analytical and Experimental Investigations on Mitigation of Interference in a DBF MIMO Radar. IEEE Trans. Microw. Theory Tech. 2017, 65, 1727-1734. [CrossRef]

4. Bechter, J.; Eid, K.; Roos, F.; Waldschmidt, C. Digital beamforming to mitigate automotive radar interference. In Proceedings of the 2016 IEEE MTT-S International Conference on Microwaves for Intelligent Mobility (ICMIM), San Diego, CA, USA, 19-20 May 2016; pp. 1-4.

5. Sturdivant, R.L.; Chang, E.; Bartholomew, D.; Brown, R.A.; De Pillis-Lindheim, S.; Rohweller, J.D. Systems Engineering a Low Cost Digital Beam Formed Phased Array for IoT Connectivity. In Proceedings of the 2017 International Conference on Computational Science and Computational Intelligence (CSCI), Las Vegas, NV, USA, 14-16 December 2017; pp. 1393-1395.

6. Lee, S.; Joo, J.; Choi, J.; Kim, W.; Kwon, H.; Lee, S.; Kwon, Y.; Jeong, J.; Joo, J. W-Band Multichannel FMCW Radar Sensor with Switching-TX Antennas. IEEE Sens. J. 2016, 16, 5572-5582. [CrossRef]

7. Younis, M.; Fischer, C.; Wiesbeck, W. Digital beamforming in sar systems. IEEE Trans. Geosci. Remote. Sens. 2003, 41, 1735-1739. [CrossRef]

8. Herd, J.S.; Conway, M.D. The Evolution to Modern Phased Array Architectures. Proc. IEEE 2015, 104, 519-529. [CrossRef]

9. Talisa, S.H.; O'Haver, K.W.; Comberiate, T.M.; Sharp, M.D.; Somerlock, O.F. Benefits of Digital Phased Array Radars. Proc. IEEE 2016, 104, 530-543. [CrossRef]

10. Lynch, J.J.; Kona, K.S.; Nagele, R.G.; Virbila, G.L.; Bowen, R.L.; Wetzel, M.D. 128 Element Coded Aperture Radar at 77 GHz. In Proceedings of the 2018 IEEE MTT-S International Conference on Microwaves for Intelligent Mobility (ICMIM), Munich, Germany, 15-17 April 2018; pp. 1-4.

11. Gaydos, D.; Nayeri, P.; Haupt, R. Experimental Comparison of Digital Beamforming Interference Cancellation Algorithms using a Software Defined Radio Array. In Proceedings of the 2019 United States National Committee of URSI National Radio Science Meeting (USNC-URSI NRSM), Boulder, CO, USA, 9-12 January 2019; pp. 1-2.

12. Diaz, J.D.; Salazar-Cerreno, J.L.; Ortiz, J.A.; Aboserwal, N.A.; Lebrón, R.M.; Fulton, C.; Palmer, R.D. A Cross-Stacked Radiating Antenna with Enhanced Scanning Performance for Digital Beamforming Multifunction Phased-Array Radars. IEEE Trans. Antennas Propag. 2018, 66, 5258-5267. [CrossRef]

13. Geng, Z.; Deng, H.; Himed, B. Adaptive Radar Beamforming for Interference Mitigation in Radar-Wireless Spectrum Sharing. IEEE Signal. Process. Lett. 2014, 22, 484-488. [CrossRef]

14. Sadhu, B.; Paidimarri, A.; Ferriss, M.; Yeck, M.; Gu, X.; Valdes-Garcia, A. A 128-element Dual-Polarized Software-Defined Phased Array Radio for mm-wave 5G Experimentation. In Proceedings of the 2nd ACM Workshop on Millimeter Wave Networks and Sensing Systems—mmNets '18, New Delhi, India, 29 October 2018; pp. 21-25. 
15. Johnson, M.; Dascuru, A.; Zhan, K.; Galioglu, A.; Adepu, N.; Jain, S.; Krishnaswamy, H.; Natarajan, A. A 4-element $28 \mathrm{GHz}$ Millimeter-wave MIMO Array with Single-wire Interface using Code-Domain Multiplexing in $65 \mathrm{~nm}$ CMOS. In Proceedings of the 2019 IEEE Radio Frequency Integrated Circuits Symposium (RFIC), Boston, MA, USA, 2-4 June 2019; pp. 243-246.

16. Pulipati, S.; Ariyarathna, V.; De Silva, U.; Akram, N.; Alwan, E.; Madanayake, A.; Mandal, S.; Rappaport, T.S. A Direct-Conversion Digital Beamforming Array Receiver with $800 \mathrm{MHz}$ Channel Bandwidth at $28 \mathrm{GHz}$ using Xilinx RF SoC. In Proceedings of the 2019 IEEE International Conference on Microwaves, Antennas, Communications and Electronic Systems (COMCAS), Tel-Aviv, Israel, 4-6 November 2019; pp. 1-5.

17. Yang, B.; Yu, Z.; Lan, J.; Zhang, R.; Zhou, J.; Hong, W. Digital Beamforming-Based Massive MIMO Transceiver for 5G Millimeter-Wave Communications. IEEE Trans. Microw. Theory Tech. 2018, 66, 3403-3418. [CrossRef]

18. Yu, Y.; Hong, W.; Jiang, Z.H.; Zhang, H.; Guo, C. Multibeam Generation and Measurement of a DDS-Based Digital Beamforming Array Transmitter at Ka-Band. IEEE Trans. Antennas Propag. 2019, 67, 3030-3039. [CrossRef]

19. Wu, Y.M.; Ke, C.-Y.; Wang, C.C.; Tang, Y.H.; Chen, Y.-W.; Li, C.-T.; Chang, L.-H.; Chu, C.-Y.; Su, B.; Chu, T.-S.; et al. An X-band Scalable $4 \times 4$ Digital Phased Array Module using RF SoC and Antenna-in-Package. In Proceedings of the 2019 IEEE Radar Conference (RadarConf), Boston, MA, USA, 22-26 April 2019; pp. 1-6.

20. Peng, Z.; Ran, L.; Li, C. A 24-GHz low-cost continuous beam steering phased array for indoor smart radar. In Proceedings of the 2015 IEEE 58th International Midwest Symposium on Circuits and Systems (MWSCAS), Fort Collins, CO, USA, 2-5 August 2015; pp. 6-9.

21. Wei, Z.; Ng, D.W.K.; Yuan, J. Beamwidth Control for NOMA in Hybrid mmWave Communication Systems. In Proceedings of the ICC 2019-2019 IEEE International Conference on Communications (ICC), Shanghai, China, 20-24 May 2019; pp. 1-6.

22. Zhang, R.; Zhou, J.; Lan, J.; Yang, B.; Yu, Z. A High-Precision Hybrid Analog and Digital Beamforming Transceiver System for 5G Millimeter-Wave Communication. IEEE Access 2019, 7, 83012-83023. [CrossRef]

23. Xiong, W.; Lu, J.; Tian, X.; Chen, G.; Pham, K.; Blasch, E. Cognitive radio testbed for Digital Beamforming of satellite communication. In Proceedings of the 2017 Cognitive Communications for Aerospace Applications Workshop (CCAA), Cleveland, OH, USA, 27-28 June 2017; pp. 1-5.

24. Arruela, R.; Marinho, D.; Varum, T.; Matos, J.N. A Ka-band Frontend for mmWave MIMO and Beam forming Applications. In Proceedings of the IEEE MTT-S International Microwave and Optoelectronics Conference (IMOC), Aveiro, Portugal, 10-14 November 2019; pp. 1-3. Available online: https://www.it.pt/Publications/ DownloadPaperConference/35051 (accessed on 14 October 2020).

25. Marinho, D.; Arruela, R.; Varum, T.; Matos, J.N. Application of Digital Beamforming to Software Defined Radio 5G / Radar Systems. In Proceedings of the IEEE MTT-S International Microwave and Optoelectronics Conference (IMOC), Aveiro, Portugal, 10-14 November 2019; pp. 1-3. Available online: https://www.it.pt/ Publications/DownloadPaperConference/35052 (accessed on 14 October 2020).

26. Varum, T.; Ramos, A.; Matos, J.N. Planar microstrip series-fed array for 5G applications with beamforming capabilities. In Proceedings of the 2018 IEEE MTT-S International Microwave Workshop Series on 5G Hardware and System Technologies (IMWS-5G), Dublin, Ireland, 30-31 August 2018; pp. 1-3.

27. Balanis, A.C. Antenna Theory Analysis and Design, 4th ed.; John Wiley \& Sons: New York, NY, USA, 2016.

Publisher's Note: MDPI stays neutral with regard to jurisdictional claims in published maps and institutional affiliations.

(C) 2020 by the authors. Licensee MDPI, Basel, Switzerland. This article is an open access article distributed under the terms and conditions of the Creative Commons Attribution (CC BY) license (http://creativecommons.org/licenses/by/4.0/). 